\title{
The Performance Comparison of the Channel Estimation Methods Based on Pilots Symbol Interpolation in HF OFDM System
}

\author{
Tuo Xin-yu ${ }^{1, a}$, ,CHENG Wei ${ }^{1, b}, Y A N G$ Rui-juan ${ }^{1, c}$ Cheng Hong-bin ${ }^{1, d}$ \\ ${ }^{1}$ Wuhan Electronic Information Academy, Wuhan City, Hubei Province, China \\ a Tuoxinyu2009@qq.com, ${ }^{\text {b } C h w 7711 @ s i n a . c o m, ~}{ }^{c}$ ruijuany@sohu.com, ${ }^{d} 13297979388 @ 163 . c o m$
}

Keywords: High Frequency Communication, Orthogonal Frequency Division Multiplexing (OFDM); Channel Estimation; Pilot Symbol Interpolation

Abstract. In the field of the application of modern HF communication, much more attention is paid to the technology of HF OFDM modulation-demodulation. HF channel is the frequency and time selective fading channel. To reliably receive information, HF receiver must estimate real-time channel parameters to equalize. A performance comparing with the channel parameters estimate methods based on the first-order linear interpolation, Gaussian interpolation, Cubic interpolation and DFT interpolation is analyzed. Simulation results show that a better performance will gain when using DFT interpolation channel estimate method in HF OFDM system.

\section{Introduction}

HF communication plays an important role in the field of modern remote communication. With the advantages of anti-multipath time-delay broadening of HF channel effectively and equalizing simply, the technology of HF OFDM modulation-demodulation has gradually been the research hotspot of modern HF communication[1,2,3]. Due to the effect of multipath fading and Doppler frequency shift, HF channel is the frequency and time selective fading channel. To reliably receive information, HF receiver must estimate real-time channel parameters. For adapting the time-varying characteristic of radio channel, in OFDM system, an effective method is to insert pilot Symbols in data stream of the transmitter, and then get the channel response of pilots position by extracting these pilots in the receiver, finally estimate the channel response of other carriers position by using interpolator.

LS[4] or MMSE[5] Channel Estimation method can be used to estimate the channel response of pilots position. However, MMSE channel estimation method need to know the statistical characteristics of the channel, and large amount of calculation for existing matrix inversion operation, which is difficult to be applied in real systems. LS channel estimation method is widely used because of its simplicity. The channel interpolation algorithms used commonly are the first-order linear interpolation[6], Gaussian interpolation[7], Cubic interpolation[8] and DFT interpolation[9]. In this paper, a performance comparing of the OFDM system with above algorithms based on the LS method to estimate pilot channel is analyzed, and to provide a basis for selecting the channel estimation method.

\section{The channel estimation model based on pilot symbol interpolation in OFDM system}

In the OFDM system, the linear convolution of the signal $x(n)$ and the impulse response of the channel is converted into cyclic convolution due to the addition of cyclic prefix. When the length of 
the cyclic prefix is greater than the maximum time delay spread, the response $y(n)$ of the channel to can be represented by the cyclic convolution of $x(n)$ and $h(n)$, that is

$$
y(n)=x(n) \otimes h(n)+v(n) .
$$

Where $0 \leq n \leq N_{s}-1, N_{s}$ is the OFDM symbol length, $v(n)$ is the system noise. The frequency domain expression of formula (1) is given by

$$
Y(k)=X(k) H(k)+N(k) .
$$

Where $0 \leq k \leq N_{s}-1$, respectively, $Y(k), X(k), H(k), N(k)$ is the frequency domain expression of $y(n), x(n), h(n), v(n)$. If the estimate $\hat{H}(k)$ of $H(k)$ is known, the estimate $\hat{X}(k)$ of $X(k)$ can be obtained by

$$
\hat{X}(k)=\frac{Y(k)+N(k)}{\hat{H}(k)}=X(k) \frac{H(k)}{\hat{H}(k)}+\Theta(k) .
$$

Where $\Theta(k)$ is the estimated error component caused by the noise. If $\hat{H}(k)$ is unbiased estimate and $N(k)$ is zero mean white noise process, then $\hat{X}(k)$ is also unbiased. Thus it is essential for the demodulation of $\hat{X}(k)$ by obtaining accurate channel estimate $\hat{H}(k)$.

The channel estimation model based on pilot symbol interpolation is shown in figure 1.

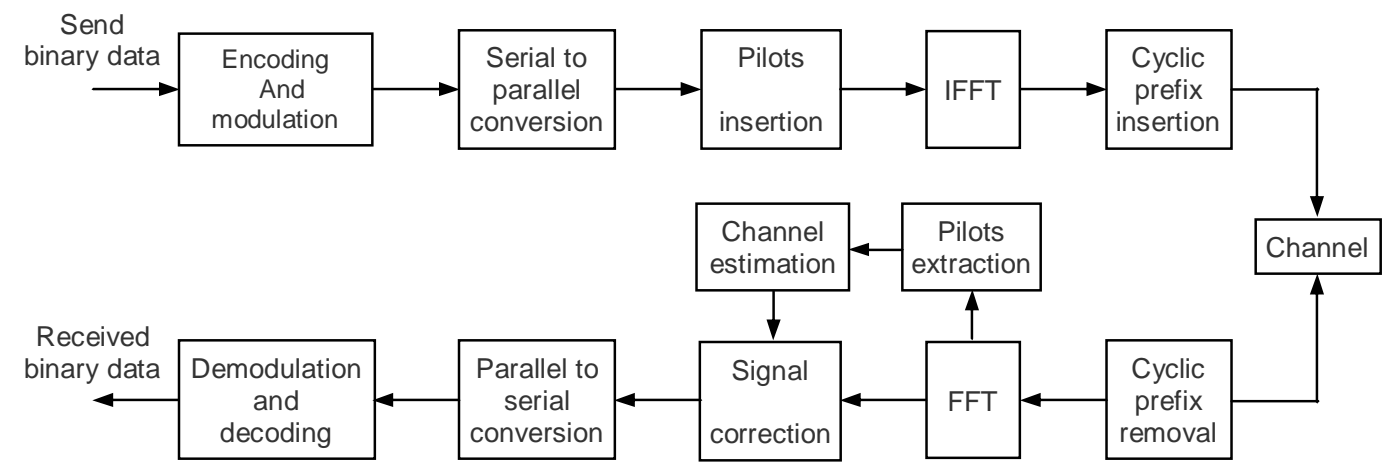

Fig.1 Block diagram of the channel estimation model based on pilot symbol interpolation

Assuming that the pilots number is $N_{p}$, the transmitted pilots interval is $L=N_{s} / N_{p}$, transmitted pilots symbols is

$$
\mathbf{X}_{p}=\left[X_{p}(0), X_{p}(1), \mathrm{L}, X_{p}\left(N_{p}-1\right)\right]
$$

And the received pilot signal is $\mathbf{Y}_{p}=\left[Y_{p}(0), Y_{p}(1), \cdots, Y_{p}\left(N_{p}-1\right)\right]$, we can get the channel frequency responses $\hat{H}_{p}(k)$ of the pilots according to some estimation criterion, then the total response $\hat{H}(k)$ of the channel can be obtained by $\hat{H}_{p}(k)$ interpolation. Because the channel response of the data carriers is obtained by the interpolation of the channel response of adjacent pilot carriers, the performance of channel estimation based on pilot interpolation is largely dependent on the accuracy of the channel estimation and the validity of the interpolation method. 


\section{The channel estimation methods based on pilot symbol interpolation}

After obtaining the channel response of comb pilots, the OFDM system receiver calculates the channel estimation values of all data subcarriers by interpolation processing of channel estimation values on pilots, then achieves frequency-domain equalization. The channel interpolation algorithms used commonly are the first-order linear interpolation, Gaussian interpolation, Cubic interpolation and DFT interpolation.

(1) The first-order linear interpolation algorithm

The basic principle of the linear polynomial interpolation algorithm is to approximate the value of the interpolation point by using piecewise continuous curve. The first order linear polynomial interpolation algorithm determines the frequency response of data carriers between two pilots by using two adjacent pilot valuations, the expression is as follows

$$
\hat{H}(k)=\hat{H}(m L+l)=\left(1-\frac{l}{L}\right) \hat{H}_{p}(m L)+\frac{l}{L} \hat{H}_{p}((m+1) L) \quad 0 \leq l \leq L-1,0 \leq m \leq N_{p}-1 .
$$

Where $L$ is the pilots interval, $N_{p}$ is the pilots number, $\hat{H}_{p}(m L)$ is the values of channel response estimated by pilots.

(2) Gaussian interpolation algorithm

Gauss interpolation need three pilot signals to estimate, which can provide better performance in the case of complexity not too much. Gauss interpolation formula in frequency domain is given by

$$
\hat{H}(k)=\hat{H}(m L+l)=C_{1} \hat{H}_{p}((m-1) L)+C_{0} \hat{H}_{p}(m L)+C_{-1} \hat{H}_{p}((m+1) L) .
$$

Where $C_{1}=\frac{\alpha(\alpha-1)}{2}, C_{0}=-(\alpha-1)(\alpha+1), C_{-1}=\frac{\alpha(\alpha+1)}{2}, \alpha=l / L$, with $0 \leq l \leq L-1$.

Gauss interpolation is actually a curve fitting, its estimation precision is generally superior to the linear interpolation of the line fitting. So the linear interpolation can be regarded as a special case of Gauss interpolation.

(3) Cubic interpolation algorithm

Cubic spline curve is a piecewise curve, thus it can reflect the change trend of real curves better. The three order curve equation between each pair of $\left(m L, H_{p}(m L)\right)$ and $\left((m+1) L, H_{p}((m+1) L)\right)$ is as follows

$$
H_{m}(k)=a_{m}(k-m L)^{3}+b_{m}(x-m L)^{3}+c_{m}(x-m L)+d_{m}, 0 \leq m \leq N_{c} .
$$

Where $N_{c}$ is the number of segments with $N_{c}+1$ points in total. If the number of pilots $N_{p}$ is even, then $N_{c}=N_{p}$, and $N_{p}$ is odd, then $N_{c}=N_{p}-1$. The following conditions must be met for a piecewise function

$$
\begin{gathered}
H_{m}(m L)=H_{p}(m L) . \\
H_{m}((m+1) L)=H_{p}((m+1) L) .
\end{gathered}
$$

In addition, in order to make the interpolation process smoother, the first order and the second order derivative of the curve must be continuous, that is

$$
\begin{aligned}
& H_{m-1}^{\prime}(m L)=H_{m}^{\prime}(m L), m=1,2, \ldots, N_{c}-1 . \\
& H_{m-1}^{\prime \prime}(m L)=H_{m}^{\prime \prime}(m L), m=1,2, \ldots, N_{c}-1 .
\end{aligned}
$$

From the upper equations, we get 


$$
\begin{gathered}
a_{m}=\frac{1}{\Delta x_{m}^{2}}\left(-2 \frac{\Delta y_{m}}{\Delta x_{m}}+H_{p}^{\prime}(m L)+H_{p}^{\prime}((m+1) L)\right), m=0,1, \ldots, N_{C}-2 . \\
b_{m}=\frac{1}{\Delta x_{m}}\left(3 \frac{\Delta y_{m}}{\Delta x_{m}}-2 H_{p}^{\prime}(m L)-H_{p}^{\prime}((m+1) L)\right), m=0,1, \ldots, N_{C}-2 . \\
c_{m}=H_{p}^{\prime}(m L), m=0,1, \ldots, N_{c}-1 . \\
d_{m}=H_{p}(m L), m=0,1, \ldots, N_{c}-1 .
\end{gathered}
$$

Where $\Delta x_{m}=(m+1) L-m L=L, \Delta y_{m}=H_{p}((m+1) L)-H_{p}(m L)$.

(4) DFT interpolation algorithm

On the basis of the DFT nature, if the value of points behind $N-M$ is 0 in the sequence of $N$ points, the values of Fourier transform at $N / M$ 's integer multiple are same as frontal $M$ points of the sequence, and the values of Fourier transform at $N / M$ 's non integer multiple is a linear combination of Fourier transform of the truncated sequences. The channel estimation method based on DFT restores the frequency response of the channel mainly by the principle, which zero-fill in time domain is equivalent to interpolation in frequency domain in signal processing.

In DFT interpolation, the pilots will be transformed to the time domain by IFFT at first, that is

$$
\left[h_{0}, h_{1}, \ldots, h_{N_{p}-1}\right]=\operatorname{IFFT}\left[H_{0}, H_{1}, \ldots, H_{N_{p}-1}\right] .
$$

Then the frequency response of the channel at each subcarriers can be obtained by transforming the impulse response of the time domain with zero-fill to the frequency domain, that is

$$
\left[\hat{H}_{1}, \hat{H}_{2}, \ldots, \hat{H}_{N}\right]=\operatorname{FFT}\left[h_{0}, h_{2}, \ldots, h_{N_{p}-1}, 0, \ldots, 0\right]_{N} .
$$

\section{Simulation and analysis}

In this section, a performance comparing with the channel parameters estimate methods based on the first-order linear interpolation, Gaussian interpolation, Cubic interpolation and DFT interpolation in HF OFDM system is simulated and analyzed respectively. The simulation conditions are as follows: There are 128 subcarriers with the interval of $46 \mathrm{~Hz}, 112$ data subcarriers, 16 comb pilots which are inserted uniformly with the interval of 8 and the position of $8 k(0 \leq k \leq 15)$, 6 multipaths whose power obeys exponential distribution. Moreover, we assume the system which adopts BPSK modulation and is an ideal synchronization.

Fig. 2 shows the simulation results of the channel estimation mean square error(MSE) of different interpolation methods. From Fig. 2 we know, the channel estimation MSE of the first order linear interpolation, Gauss interpolation, Cubic interpolation is little different with DFT interpolation in the case of low signal to noise ratio(SNR); and with the increase of SNR, the first order linear interpolation, Gauss interpolation, Cubic interpolation produce "error floor phenomenon", but DFT interpolation no; then when the SNR is greater than $26 \mathrm{~dB}$, the performance of the DFT interpolation method is better than the other three methods obviously. 


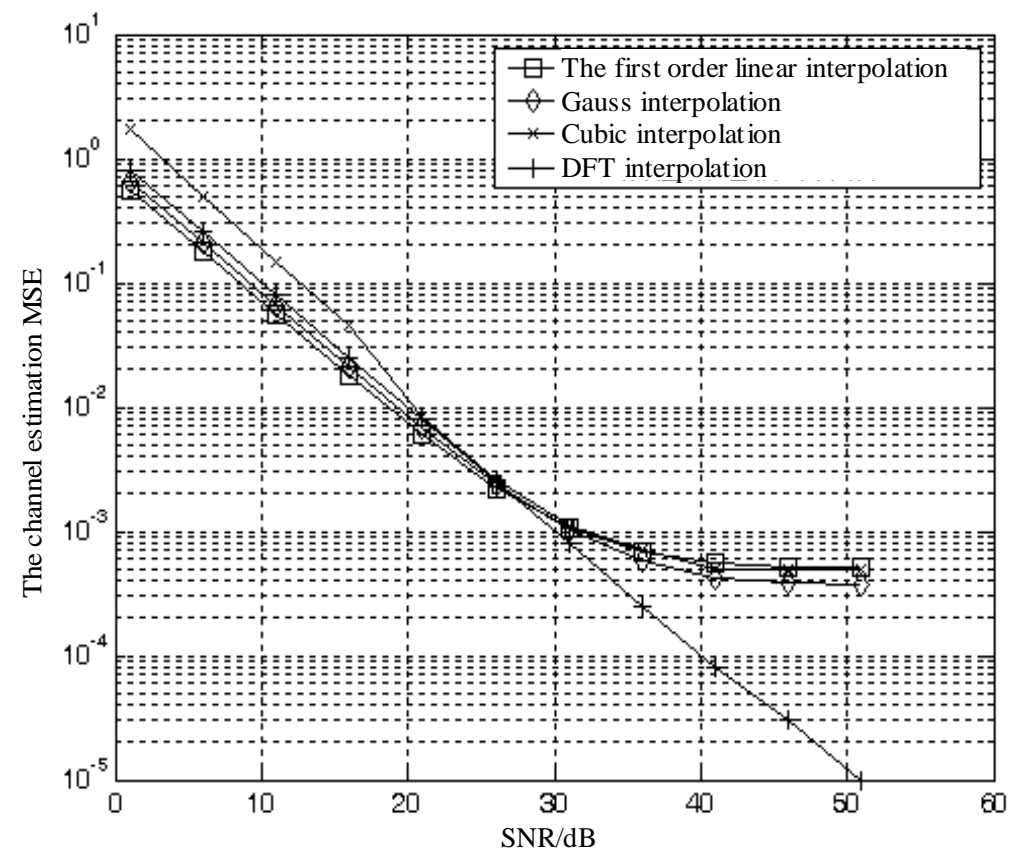

Fig. 2 The channel estimation MSE of different interpolation methods

(6 multipaths, the interval of pilots is 8)

Fig. 3 shows the simulation results of the bit error rate(BER) performance that the HF OFDM system is equalize by using the channel estimation values of different channel interpolation methods and the ideal channel values. From Fig. 3 we know, the BER performance of the ideal channel is the best, the BER of the first order linear interpolation, Gauss interpolation, Cubic interpolation is very similar with DFT interpolation in the case of low signal to noise ratio(SNR), which is unified with the result of MSE, and the smaller the channel estimation MSE is, the lower the BER of the system by equalizing is; and with the increase of SNR, the BER of the first order linear interpolation, Gauss interpolation, Cubic interpolation produce also "error floor phenomenon" with "error floor phenomenon" of the channel estimation MSE; then when the SNR is greater than 41dB, the BER performance of the DFT interpolation

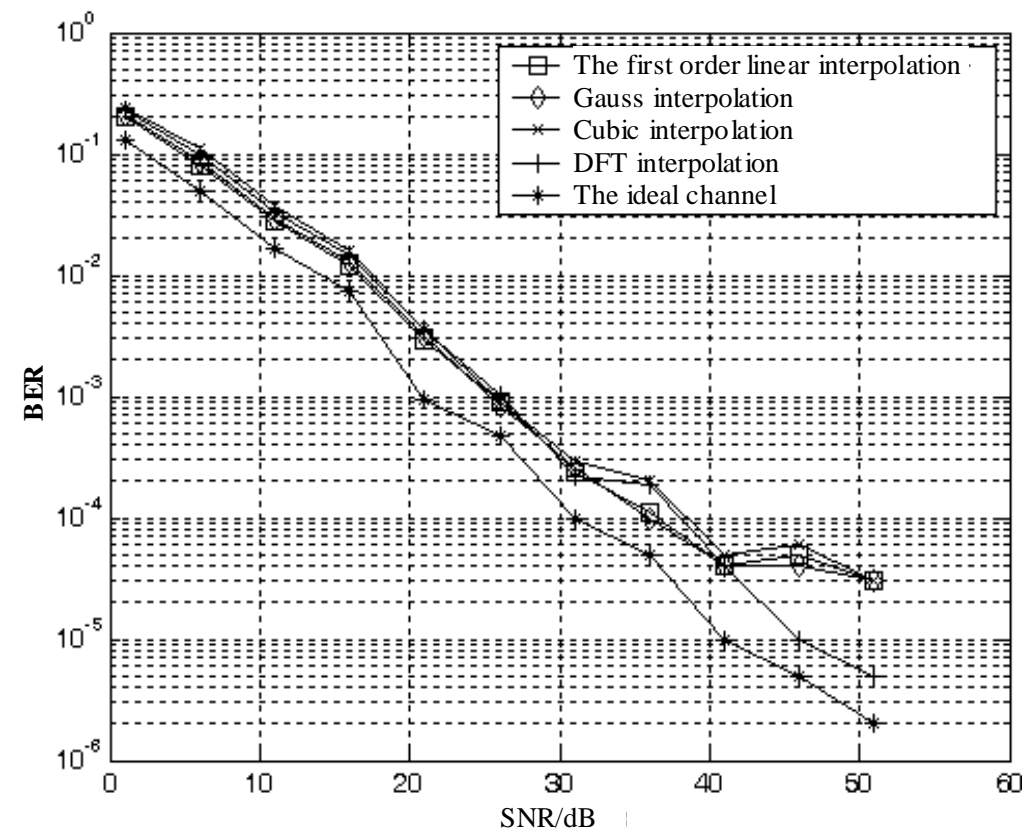

Fig. 3 The BER performance of different interpolation methods

(6 multipaths, the interval of pilots is 8) 
In order to study the effect of the number of multipaths on performance of the channel interpolation estimation algorithm, Fig. 4, 2, 5 show respectively the simulation results of the channel estimation MSE of different interpolation methods with 3, 6, 9 multi-paths and same other conditions as above. From Fig. 4, 2, 5 we know, the estimation performance of various interpolation methods with different multipaths show better consistent; but the first order linear interpolation, Gauss interpolation and Cubic interpolation method are more sensitive to the number of multipaths because not only their "error floor value" of the channel estimation MSE increases but also their SNR decreases when "error floor phenomenon" comes into being with the increase of multipaths; then DFT interpolation method shows good performance which its channel estimation MSEs with different multipaths are very close as well as it doesn't produce "error floor phenomenon".

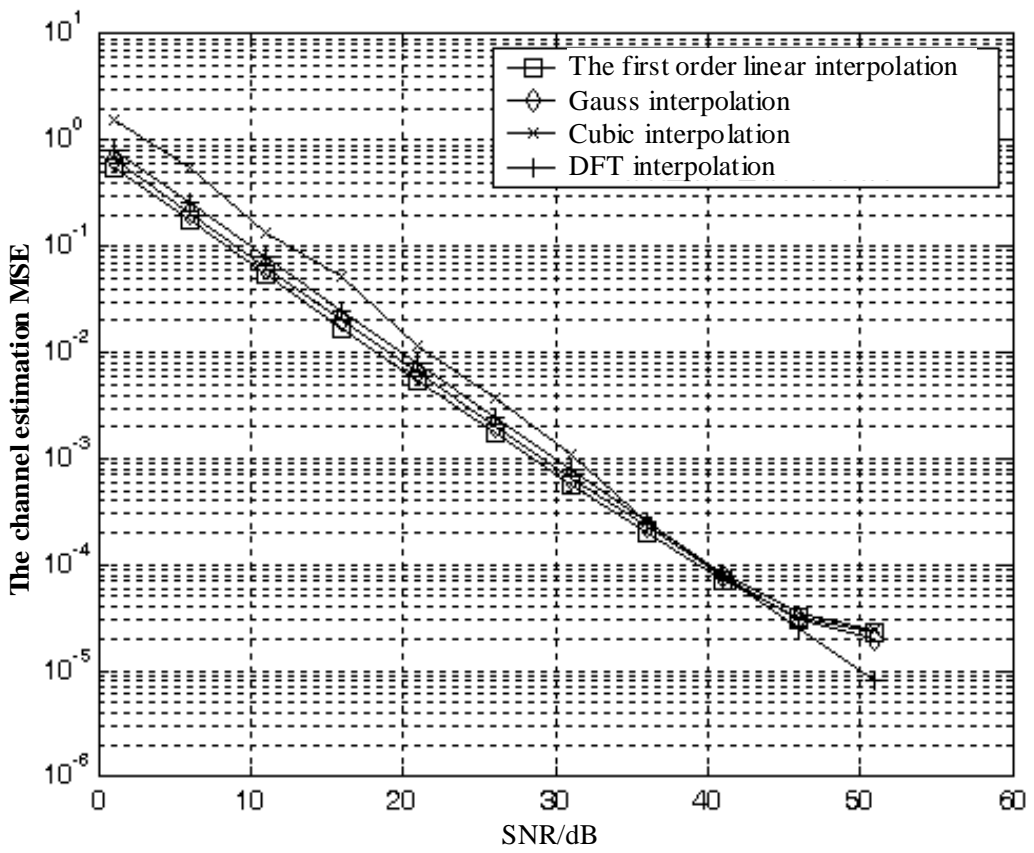

Fig. 4 The channel estimation MSE of different interpolation methods (3 multipaths, the interval of pilots is 8)

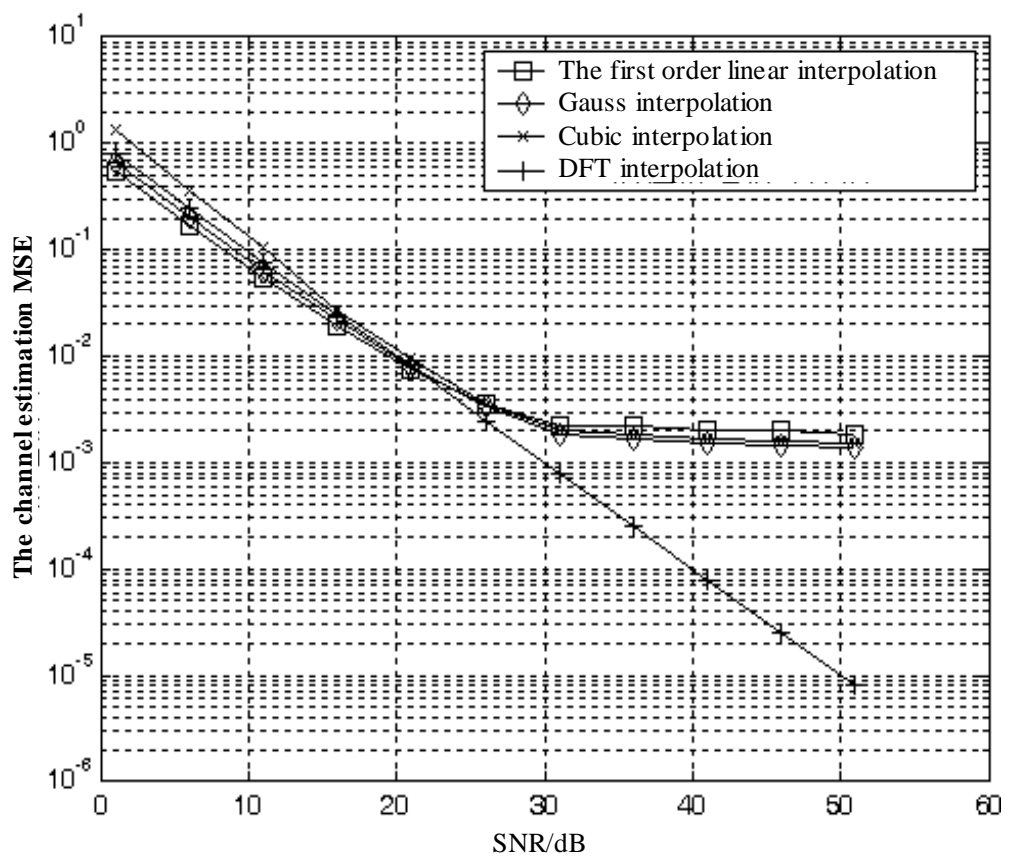

Fig. 5 The channel estimation MSE of different interpolation methods (9 multipaths, the interval of pilots is 8) 
In order to study the effect of the interval of pilots on performance of the channel interpolation estimation algorithm, Fig. 6, 2, 7 show respectively the simulation results of the channel estimation MSE of different interpolation methods whose pilots are inserted uniformly with the interval of 4, 8 , 16(means the number of pilots is respectively 32,16,8), and with 6 multi-paths and same other conditions as above. From Fig. 6, 2, 7 we know, reducing the interval of pilots which is equivalent to increase the number of pilots can decrease the "error floor value" of the channel estimation MSE in the first order linear interpolation, Gauss interpolation and Cubic interpolation method, and vice versa; but the change of the interval of pilots has little effect on the performance of DFT interpolation. So in the actual low SNR system, DFT interpolation method can be used to improve the transmission efficiency by suitably reducing the number of pilots on the condition of ensuring the pilots insertion interval.

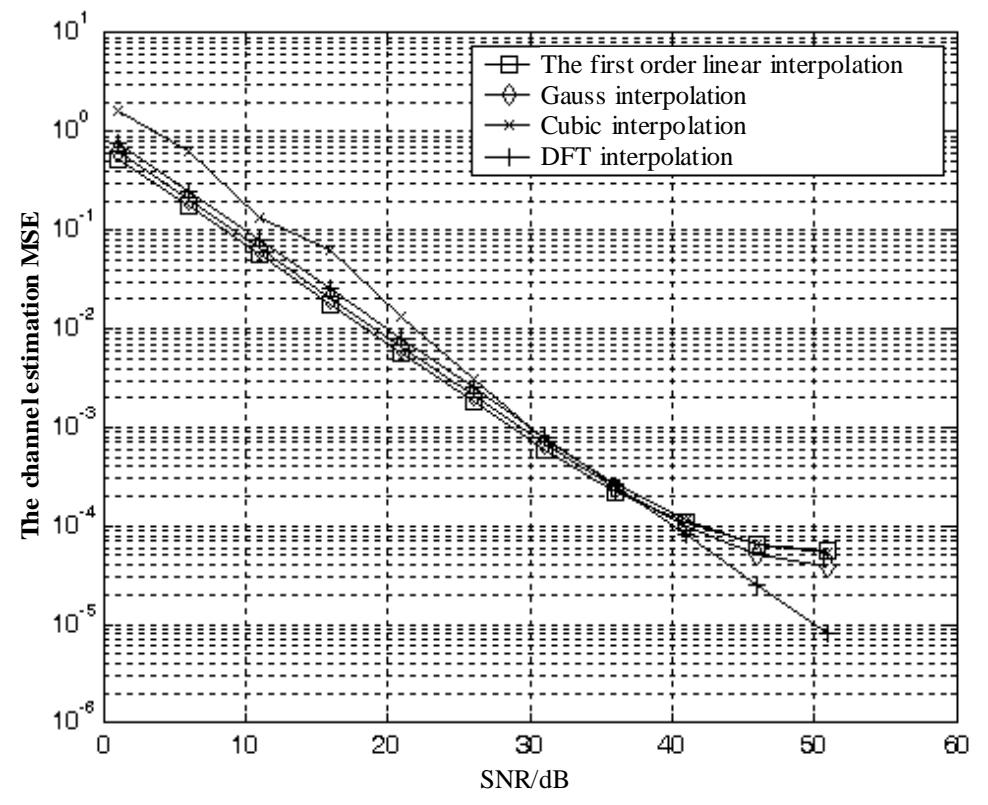

Fig. 6 The channel estimation MSE of different interpolation methods (6 multipaths, the interval of pilots is 4 )

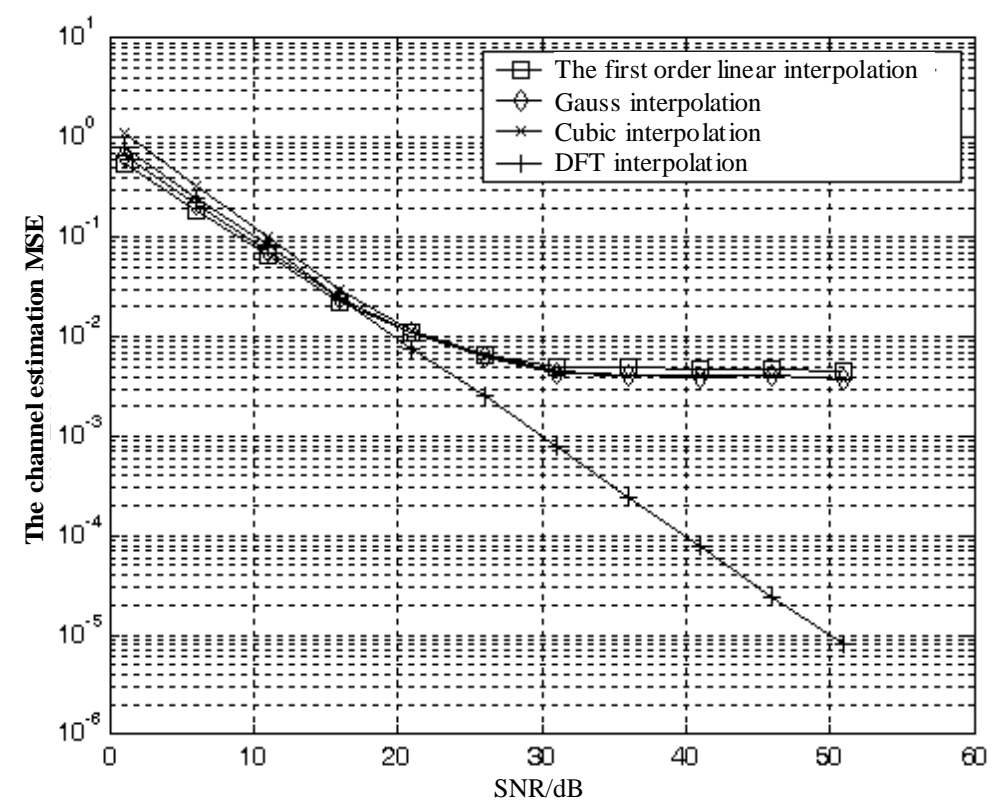

Fig. 7 The channel estimation MSE of different interpolation methods (6 multipaths, the interval of pilots is 16) 


\section{Conclusions}

Channel error can cause the loss of SNR of HF OFDM system, and decrease its performance. The channel estimation methods based on pilots symbol interpolation, by which get the frequency response of the channel at pilots position at first, then estimate the frequency response at each subcarriers position by using interpolator, is widely used to estimate various time-varying channels parameters . In this paper, the performance of the first order linear interpolation, Gauss interpolation, Cubic interpolation, interpolation and DFT interpolation method used in the channel parameters estimation under different conditions of HF OFDM system is analyzed and compared by computer simulation. The results show, the channel estimation MSE of the first order linear interpolation, Gauss interpolation, Cubic interpolation method produce "error floor phenomenon" due to the effect of the InterSymbol Interference(ISI) caused by multipaths, but DFT interpolation method may be applied to estimate the channel parameters of HF OFDM system for its better performance than the above three methods.

\section{References}

[1] M.Coutolleau. New studies about a high data rate HF parallel modem[C]. Proceedings of IEEE Military Commun.,1998(2), pp. 381-385.

[2] S.Zazo. High frequency data link(HFDL) for civil aviation: a comparison between single and multitone voiceband modems[C].Proceedings of IEEE VTC'99, 1999, pp. 2113-2118.

[3] Liu Wei, Zhang Hai-lin, Liu Zeng-ji. Design of a wideband HF data modem Based OFDM[J]. Systems Engineering and Electronics, Vol.25,No.12,2003, pp. 1565-1568.

[4] Ahmad R.S.Bahai, B.R.Saltzberg. Multi-Carrier Digital Communication Theory and Application of OFDM[M]. Kluwer Academic/Plenum Publisher, Newyork, 1999.

[5] B.Muquet, Z.Wang, G.B.Giannakis, M.de Courville. Cyclic Prefixing or Zero Padding for Wireless Multicarrier Transmissions[J]. Communications, IEEE Transactions on, Vol.50, No.12, Dec.2002, pp. 2136-2148.

[6] Wang Qin. Study on synchronization and equalization technology of HF OFDM system[D]. Master Dissertation, The PLA Information Engineering University, 2007

[7] Moon J K,Choi S I. Performance of channel estimation methods for OFDM systems in a multipath fading channels[J]. IEEE Transaction on Consumer Electronics, February 2000, 46(1), pp. 161-170.

[8] Fritsch, Carlson R. Monotone piecewise cubic interpolation[J]. SIAM J. Numerical Analysis, 1980, 17(2), pp. 238-246.

[9] Fenrandez-Getino Gareia, M.J.Paez-Borrallo, J.M.Zazo,S. DFT-based channel estimation in 2D-Pilot-symbol-aided OFDM wireless systems[J]. Vehicular Technology Conference,2001. VTC 2001 Spring. IEEE VTS 53rd,Vol,2,6-9 May 2001, pp. 810-814. 